\title{
Optimal Phase Swapping in Low Voltage Distribution Networks based on Smart Meter Data and Optimization Heuristics
}

\author{
Izaskun Mendia $^{1}$, Sergio Gil-López ${ }^{1}$, Javier Del Ser ${ }^{1,2,3}$, Ana Gonzalez \\ Bordagaray $^{3}$, Jesús Garcia Prado ${ }^{3}$, and Manuel Vélez ${ }^{2}$ \\ 1 TECNALIA, E-48160 Derio, Spain, \\ \{izaskun.mendia, sergio.gil, javier.delser\}@tecnalia.com \\ 2 University of the Basque Country UPV/EHU, 48013 Bilbao, Spain, \\ $\{$ javier.delser, manuel.velez\}@ehu.eus \\ 3 Basque Center for Applied Mathematics (BCAM), 48009 Bilbao, Spain \\ 4 IBERDROLA Distribucion Electrica, S. A., 48003 Bilbao, Spain, \\ \{ana.gb,jgarciapr\}@iberdrola.es
}

\begin{abstract}
In this paper a modified version of the Harmony Search algorithm is proposed as a novel tool for phase swapping in Low Voltage Distribution Networks where the objective is to determine to which phase each load should be connected in order to reduce the unbalance when all phases are added into the neutral conductor. Unbalanced loads deteriorate power quality and increase costs of investment and operation. A correct assignment is a direct, effective alternative to prevent voltage peaks and network outages. The main contribution of this paper is the proposal of an optimization model for allocating phases consumers according to their individual consumption in the network of low-voltage distribution considering mono and bi-phase connections using real hourly load patterns, which implies that the computational complexity of the defined combinatorial optimization problem is heavily increased. For this purpose a novel metric function is defined in the proposed scheme. The performance of the HS algorithm has been compared with classical Genetic Algorithm. Presented results show that HS outperforms GA not only on terms of quality but on the convergence rate, reducing the computational complexity of the proposed scheme while provide mono and bi phase connections.
\end{abstract}

Keywords: Smart Meter; Load Curve; Harmony Search

\section{Introduction}

In the electrical distributions systems of most utilities over the world, three phases of alternate current are utilized at each feeder aimed at increasing the energy efficiency in low-voltage distribution networks. During the past decades a huge increment in housing construction has occurred in many countries (especially in Spain), which has given rise to highly-populated low-voltage distribution 
networks. This noted fact, combined with the ever-growing use of home electronic devices, has heretofore unchained a dramatically sharp load growth. When held together, these two key points lay an operational challenge to distribution companies because the so-called feeder tripping problem [1] is even more involved due to in-excess neutral current caused by the unbalance loads among the three phases. Unbalanced loads between phases may lead to undesirable situations, including [2]: current increase in the most heavily loaded phase limits the amount of power transferred on a feeder; current increment in the neutral conductor; and problems with voltage drops in phase with higher loads, which ultimately results in a low quality of service. As a result, power losses in distribution networks may vary significantly depending on the load imbalance.

Accordingly, if loads on each phase are properly balanced technical losses will be notably reduced [3-5]. Consequently, a proper balance between the three phases will contribute to: 1) an optimized network infrastructure by increasing the capacity of distribution feeders, hence avoiding the deployment of unnecessary extra feeders and consequently reducing distribution costs; 2) a reduced monitoring complexity of the low-voltage distribution network due to the reduction of instabilities generated by the presence of high currents in the neutral conductor; and 3) an improved voltage profile due to the homogenization of the voltage drops at each stage of the distribution line.

There are two major techniques for phase balancing in the related literature [2]: feeder reconfiguration at the system level, and phase swapping at the feeder level. The former is a process of changing the topological structure of the distribution systems by altering the open/closed status of single phase sections and tie switches [6]. In phase swapping, however, the objective is to determine to which phase each load should be connected in order to reduce the unbalance when all phases are added into the neutral conductor. This objective can be formulated as a combinatorial optimization problem with exponential complexity growth with the number of possible loads: for the case of $N$ loads to be connected to 6 phases ( 3 single phases and 3 complex phases), there is a total of $6^{N}$ combinations (possible solutions).

Many research contributions in the last years have dealt with the phase swapping problem [7], each resorting to different approaches and diverse methodologies such as Simulated Annealing [8-10], Neural Networks [5, 11], Genetic Algorithms [12], Tabu Search [13], Greedy approaches and Dynamic Programming [2], among others. In [14] phase swapping is addressed as a load-to-line assignment problem and tackled under a mixed-integer programming formulation. In [15] the optimal load phase balance is obtained by solving the load redistribution problem by using a decaying self-feedback continuous Hopfield neural network (ADSCHNN). Likewise the work in [16] proposes a new approach for phase balancing planning using a specialized Genetic Algorithm which considers discretized load duration curve.

To the knowledge of the authors none of the above references accounts for two practical situations of real low-voltage distribution networks. The first one relates to the use of the information provided by Advanced Metering Infrastruc- 
tures (AMIs) or Smart Meters, which provides a deeper, fine-grained knowledge of load patterns, far beyond the coarse-grained monitoring performed until their appearance. Indeed, by virtue of the hourly load patterns provided by the AMIs not only operational costs are reduced and the quality of service is improved, but also an evidence of paramount importance for our work has been unveiled: even if phase balancing can be met over a certain time span (i.e. yearly or monthly), the characteristics of connected loads vary continuously, which causes punctual, undesired situations of unbalanced phases. The second aspect which has not been taken into consideration in the literature as mentioned in [2] is that loads can be connected to two phases, as opposed to related contributions so far which consider only single phase connections. This point increases the computational complexity of the phase swapping problem by increasing the number of possibilities by which the load could be connected, i.e. from $3^{N}$ to $6^{N}$ for $N$ loads.

The above challenges motivate the development of new heuristic procedures that efficiently tackle the phase swapping problem taking into consideration the time variance of load patterns and mono- and bi-phase connections. For this purpose in this paper we propose to apply the Harmony Search (HS [17]) algorithm as an heuristic procedure for solving the aforementioned problem. HS has been used for NP-hard problems providing a good balance between computational complexity and quality of the provided solutions $[18,19]$. This manuscript delves into the adaptation of the HS characteristics to phase swapping introducing a novel metric definition and solution encoding for bi-phase connections. This analysis will provide a realistic procedure for optimizing the topology of lowvoltage distribution networks in real Smart Grids minimizing, statistically, the load unbalance between the three phases. The performance of the proposed approach is assessed over a real use case comprising an entirely remotely-managed distribution substation, with hourly readings of 102 customers (82 residential, 5 industrial and 15 commercial) captured over a historical depth of one year, with more than 21 million watts managed during this period. As concluded from these simulations, the performance of the solver is confirmed to be promising and superior to other genetically inspired heuristics, hence paving the way towards its practical implementation in real energy distribution systems.

\section{Problem Formulation}

As mentioned in the introduction the overall goal of this research work is to develop an heuristic method for finding the mapping from loads to phases leading to a minimum imbalance between phases taking into account the hourly consumption traces provided by smart meters. A proper balance in the circuit is achieved by minimizing the Euclidean distance between residuals and phases, so that the lower the residuals are, the smaller the total imbalance of the electrical system will be. The application of these methods will focus on real hourly customers' load patterns. The periodicity at which the algorithm is executed is a strategic decision of the utility, which should consider both the operational cost of the phase reassignment and the seasonal treatment of the historical se- 
ries. The optimization objective is to minimize the sum of Euclidean distances between the three aggregated loads of the users assigned to each phase, pair by pair. Such a difference is given, for 3 phases, $N$ loads and time $t$ (i.e. an index enumerating the 24 values for each of the 365 days in a year), by

$$
Q(\mathbf{x}, t)=\sum_{(\phi, \theta) \in \mathcal{P}} \sqrt{\left(\sum_{n=1}^{N} \mathbb{I}\left(x_{n}, \phi\right) E_{n}(t)\right)^{2}-\left(\sum_{n^{\prime}=1}^{N} \mathbb{I}\left(x_{n^{\prime}}, \theta\right) E_{n^{\prime}}(t)\right)^{2}},
$$

where $\mathcal{P} \triangleq\{(R, S),(S, T),(R, T)\}, E_{n}(t)$ is the energy consumption of load $n$ at time $t, \mathbf{x} \triangleq\left\{x_{n}\right\}_{n=1}^{N}$ is the mapping from loads to phases such that, by assuming a biphasic electric network, $x_{n} \in\{R R, R S, R T, S S, S T, T T\} \forall n \in\{1, \ldots, N\}$, and $\mathbb{I}\left(x_{n}, \phi\right)$ is an indicator function taking value 1 if $\phi \in x_{n}$ and 0 otherwise. In words, each phase is represented by the sum of the hourly energy consumption of the loads assigned to that phase. The case when $Q(\mathbf{x}, t)=0$ means that the energy between phases is perfectly balanced at time $t$, hence lower values of $Q(\mathbf{x}, t)$ represent a better load balance.

Before proceeding further, it is worth to delve into the rationale why a balanced distribution network is desirable for the distributor. A perfect balance implies an electric system with minimal energy losses. Indeed, load imbalance may yield up to three times more losses through an imbalanced distribution line when compared to a balanced one. This can be argued, on the one hand, by the application of Joule's Law, which rules the conversion of energy into heat with a consequent increase in the temperature of the conductor,

$$
P=R I^{2}=\frac{E}{\Delta t}
$$

which, by applying Ohm's Law (i.e. the potential $V$ arising between the extremes of a conductor is proportional to the electric current $I$ going through it), yields

$$
V=Z I \cos \alpha \rightarrow I=\frac{E}{\Delta t V \cos \alpha}
$$

where $Z$ denotes the impedance of the conductor (line) and $\alpha$ is the angle between the current phase vector and the voltage $V$, also referred to as power factor. If we note that the current $I$ is given by the sum of the individual currents over each phase, i.e. $I=I_{R}+I_{S}+I_{T}$, a perfectly balanced line will satisfy $I_{R}=I_{S}=I_{T}=I / 3$. By contrast, an imbalanced line undergoing a strong phase imbalance with all the current circulating through a single phase fulfills that e.g. $I=I_{R}$ and $I_{S}=I_{T}=0$. Bearing this in mind, the power losses for a perfectly balanced system are given by

$$
P_{b a l}=Z\left(I_{R}^{2}+I_{S}^{2}+I_{T}^{2}\right)=Z\left(\frac{I^{2}}{9}+\frac{I^{2}}{9}+\frac{I^{2}}{9}\right)=\frac{Z I^{2}}{3},
$$

Since the load curve reports the measured energy consumption, the technical power losses $P_{b a l}$ for three phases and perfect balance are given by

$$
P_{b a l}=\frac{R E^{2}}{3(\Delta t V \cos \alpha)^{2}} .
$$


On the other hand, if we deal with an imbalanced system where all energy is conducted over a single phase, technical power losses $P_{\text {unbal }}$ will increase up to

$$
P_{\text {imbal }}=R I^{2}=\frac{R E^{2}}{(\Delta t V \cos \alpha)^{2}}=3 P_{b a l},
$$

i.e. they can potentially as high as three times the losses for the perfectly balanced case. There lies the interest of the energy distributor in balancing the consumption among phases, and the rationale for the application of the heuristic solver explained in the next section.

\section{Description of Harmony Search}

The Harmony Search is a metaheuristic algorithm based on the emulation of the music improvisation process observed in jazz bands, whose members use to combine different musical notes based on the historical record of notes played by each musician followed by an occasional, random yet slightly pitch tuning. HS maintains a pool of $K$ candidate solutions or harmonies $\left\{\mathbf{x}^{k}\right\}_{k=1}^{K}=\left\{\left\{x_{n}^{k}\right\}_{n=1}^{N}\right\}_{k=1}^{K}$, each comprising $N$ optimization variables or notes (i.e. the number of loads associated to the feeder at hand whose optimal phase assignment is to be discovered by the algorithm). The main steps of the standard HS solver [19] are as follows:

- Step 1 (initialization): this first step is only considered at the first iteration. The pool of $K$ harmonies (also referred to as Harmony Memory, HM) is initially filled, which is done uniformly at random if no a priori knowledge about the solution space is assumed. This represents the starting point for the set of candidate harmonies. For the problem at hand the alphabet of notes has 6 possible values, 3 for single-phase connections and 3 for bi-phase connections, namely $x_{n}^{k} \in\{1,2,3,4,5,6\}$ corresponding with each possible connection assignment $\{R T, R R, R S, S S, S T, T T\}$. It should be noted that the mapping from the note encoding to the sequence of phases is designed so that when tones need to be adjusted to any other tone in their vicinity, changes in the phase mapping are not drastic, as neighboring notes in the integer alphabet correspond to phases with at least one phase in common with that of the original note. In this case it is assumed that in bi-phase connections the current is equally distributed over each individual phase.

- Step 2 (Improvisation): for each of the iterations a new harmony is generated. To this end two are the operators defined in the naive HS solver:

1. Harmony Search Considering Rate (HMCR $\in \mathbb{R}[0,1]$ ), which sets the probability that the value of a new proposed note is drawn from the set of values that such a note has in the rest of harmonies, i.e. $\mathrm{HMCR}=0.9$ involves that $90 \%$ of the new notes are drawn from the whole set of harmonies at each iteration. As will be later explained, based on previous studies [20] a linear variation of the HMCR parameter along iterations has been adopted so as to enhance the convergence of the search process. Any component not selected for memory consideration will be randomly 
set to a value between the lower and upper bounds of its possible range in the defined alphabet.

2. Pitch Adjustment Rate (PAR $\in \mathbb{R}[0,1]$ ), which establishes the probability that a given note is set to one of its neighboring values within the note alphabet. i.e., PAR $=0.2$ involves that $20 \%$ of the new notes are drawn from the neighboring (lower or higher with equal probability) set of notes defined on the integer alphabet on which they are encoded.

- Step 3 (HM update): the new improvised harmony is now evaluated according to its value of the objective function which, for the case study tackled in this paper, is given by Expression (1) aggregated over a certain time horizon,

$$
f\left(\mathbf{x}^{k}\right)=\sum_{t=1}^{T} \sum_{(\phi, \theta) \in \mathcal{P}} \sqrt{\left(\sum_{n=1}^{N} \mathbb{I}\left(x_{n}^{k}, \phi\right) E_{n}(t)\right)^{2}-\left(\sum_{n^{\prime}=1}^{N} \mathbb{I}\left(x_{n^{\prime}}^{k}, \theta\right) E_{n^{\prime}}(t)\right)^{2}},
$$

where $T$ denotes the time span over which the phase balance provided by $\mathbf{x}^{k}$ is evaluated. If the objective function value for the new harmony is better (lower) than the objective function value for the worst harmony in the HM, then such a worst harmony is replaced with the newly improvised harmony.

- Step 4 (Stop criteria): if a maximum number of improvisation is reached, then stop, otherwise step 3 and step 4 are repeated.

The HMCR and PAR operators aid the algorithm in the search for better solutions, and even affect the speed of convergence. The values for these two parameters had to be optimized to find the best set of parameters in terms of balancing explorative versus exploitative character of the exploratory finding, but this study is omitted for lack of space. The work in [21] proposes to improve the performance of the HS algorithm hinges on imposing a certain progression along iterations on the values of its operational parameters of HMCR and PAR. For instance, the value of the PAR is dynamically updated according to:

$$
\mathrm{PAR}(\text { iter })=\mathrm{PAR}_{\text {min }}+\left(\mathrm{PAR}_{\text {max }}-\mathrm{PAR}_{\text {min }}\right) \cdot \xi(\text { iter })
$$

where $\mathrm{PAR}_{\text {min }}$ and $\mathrm{PAR}_{\text {max }}$ are minimum and maximum values of the adjusting rate, and $\operatorname{PAR}($ iter $)$ is the pitch adjusting rate for iteration iter $\epsilon$ $\{1,2, \ldots$, niter $\}$. The coefficient $\xi($ iter $)$ is calculated based on the iteration iter and the maximum number of iterations numMaxIter as

$$
\xi(\text { iter })=\frac{\text { iter }}{\text { numMaxIter }} .
$$

This modification of the HS algorithm, by an iterative process, establishes dynamic values for HMCR and PAR with each new iteration, seeks to avoid premature convergences in suboptimal regions. Thus, this approach prioritizes the explorative capability of the search process rather than its exploitative behavior. 


\section{Experiments and Results}

In order to evaluate the performance of the proposed scheme, a set of experiments based on real data has been designed for dealing with the phase swapping problem discussed above taking into consideration the time variance of load patterns and mono- and bi-phase connections. The data is provided by an remotely managed distribution substation. Only one feeder with three phases is considered accounting up to 102 customers, from which 82 are residential, 5 industrial and 15 commercial. One-year-long load patterns with one-hour granularity are considered in the experiments, which yields $365 \times 24$ data points per customer. The complexity of the proposed experiments is $6^{102}$ considering three monophase connections and other three bi-phase connections. The paper compares the proposed HS algorithm described in Section 3 with the Genetic Algorithm (GA) proposed in [12]. GA is controlled by mutation and crossover operators which drive the behavior of the iterative search procedure. A tournament selection is selected in an attempt at reinforcing the capacity of the GA to escape from local optima during its search. One of the most evident differences between both schemes is that GA creates new chromosomes by using only one (mutation) or two (crossover) individuals, as opposed to the HS solver which exploits the knowledge embedded in the entire set of harmonies stored in the HM.

The comparative study is discussed in statistical terms motivated by the heuristic nature of the algorithms in the benchmark and the stochasticity imposed by their probabilistic operators. A computationally fair comparison is guaranteed by setting equal the number of metric evaluations for both schemes. The values of the improvisation operators for both optimization approaches are refined based on a previous grid search not shown due to lack of space. The values for which the best balance between explorative versus exploitative behaviour of the proposed schemes was found are listed in Table 1. In order to compute performance statistics 50 Monte Carlo simulations are performed. It is important to observe in Table 1 that GA prioritizes a memory that doubles in size that of the HS approach. Therefore, for a fair comparison the maximum number of generations is set to half the one for its HS counterpart.

Table 1. Refined parameters of the HS and GA solvers.

\begin{tabular}{ccccc}
\hline No. & HS parameter & Value & GA parameter & Value \\
\hline 1 & $K$ & 50 & Population size & 100 \\
2 & {$\left[\mathrm{HMCR}_{\min }, \mathrm{HMCR}_{\max }\right]$} & {$[0.7-0.9]$} & Crossover rate & 0.9 \\
3 & {$\left[\mathrm{PAR}_{\min }, \mathrm{PAR}_{\max }\right]$} & {$[0.01-0.1]$} & Mutation rate & 0.2 \\
4 & numMaxIter $^{\text {Mum }}$ & 200 & Max. generation & 100 \\
\hline
\end{tabular}

Figure 1 shows the best metric obtained for each algorithm in the iterative process in the statistics of the 50 Monte Carlo simulations. It can be noted that HS outperforms GA, i.e. for the 50 independent runs of the algorithms the fitness values attained by HS are smaller than GA, not only in terms of its mean value but also in what regards to their dispersion (a standard deviation of $11000 \mathrm{~W}$ 
for HS and $12508 \mathrm{~W}$ for GA). Indeed only in 10 of the 50 experiments HS falls within the value range bounded as mean \pm standard deviation computed for GA. In the rest of experiments results of HS are outside this GA confidence region.

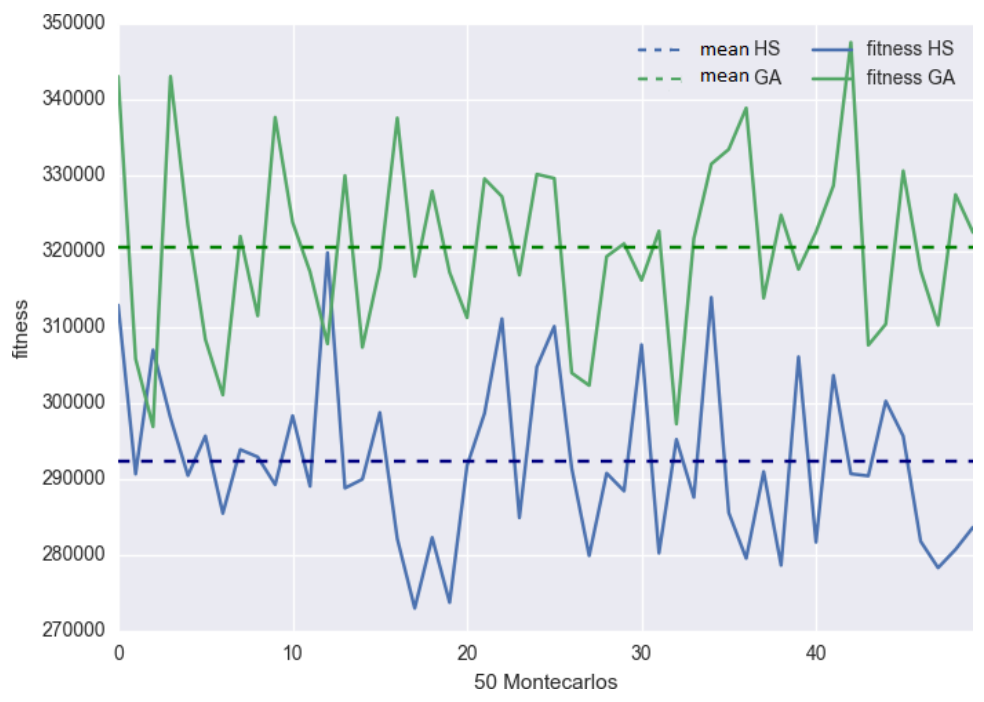

Fig. 1. Fitness value obtained over the 50 Monte Carlo experiments performed.

The discussion follows in Figure 2, where it is shown that the convergence of the proposed HS scheme is faster than that of GA. The plot depicts the average metric evolution (solid lines) throughout the 50 Monte Carlos during the iterative process of both schemes, whereas the standard deviation of the mean at each iteration is marked showing the independence of the obtained results.

The ideal case - a perfect balance between phases - is obtained when the metric as per Expression (1) is $Q(\mathbf{x}, t)=0$ at each time stamp (i.e. each hour during the whole considered year). Integrating the energy in time along the whole considered year $t \in\{1, \ldots, 365 \times 24\}$, a perfect balance means $33.3 \%$ for each phase. Figure 3 shows the integrated energy distribution per phase obtained with HS and GA; while GA renders a maximum difference of $6.8 \%$ between two phases, in the case of HS this score is limited to $4.7 \%$, which corresponds to an absolute value of $441000 \mathrm{~W}$ over the whole year. This noted fact means that there is no clear compensation of seasonality effects along the year and through users, because the hourly study shown in Figures 1 and 2 is corroborated with the yearly one in Figure 3. This does not have to be extrapolated to all situations.

\section{Conclusions and Future Research Lines}

This paper has proposed a meta-heuristic scheme specially tailored for efficiently finding the mapping from loads to phases that leads to a minimal energy imbalance between phases. The study builds upon real consumption traces hourly pro- 


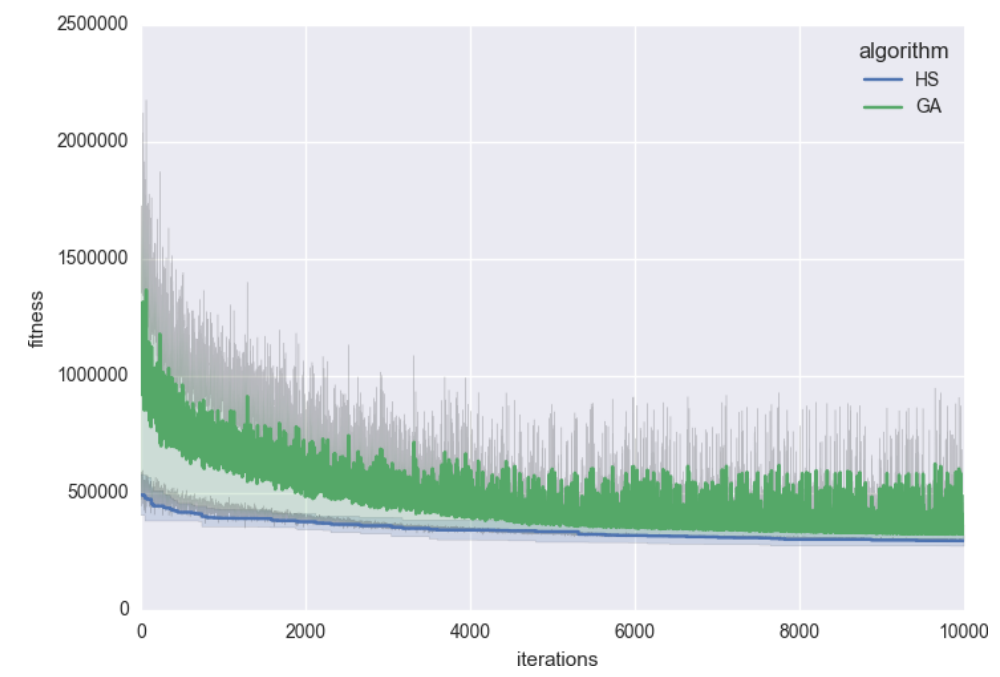

Fig. 2. Fitness convergence along iterations for both HS and GA algorithms.
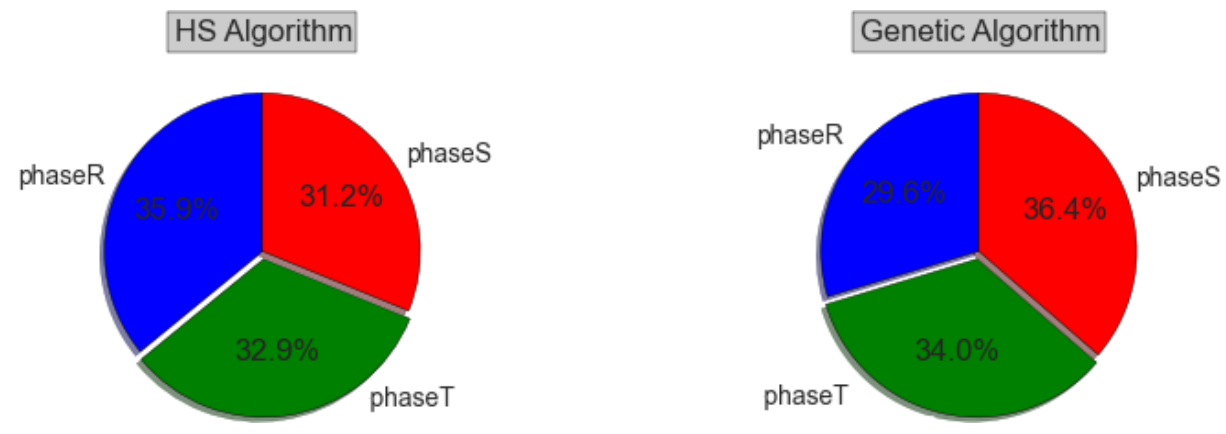

Fig. 3. Percentage distribution of energies per phase for the two algorithms.

vided by smart meters. On this purpose a novel fitness function is proposed, and modifications of the naive Harmony Search algorithm scheme are introduced. The proposed methodology is compared with a standard Genetic Algorithm (GA) from the literature, showing that the HS outperforms GA with statistical significance over 50 independent Monte Carlo experiments, not only on its hourly component but on the yearly one. Future research will focus on multi-objective formulations of this problem in order to improve further the practical benefits of the method proposed in this manuscript. The considered metrics will span beyond the reduction of the technical losses tackled in this paper towards the minimization of the operational costs derived from the reassignment of different users. In addition, the obtained conclusion - i.e. hourly results are buttressed by the yearly phase balances - will be generalized using different, more diverse substations demonstrating that hourly knowledge is necessary to avoid the seasonality compensation effect when optimizing over the whole year. 


\section{Acknowledgments}

This work was supported by the Horizon 2020 programme of the European Commission (grant 646531), as well as by the Basque Government through the ELKARTEK programme (BID3A and BID3ABI projects).

\section{References}

1. Lin Ch. H., Chen Ch. S., Chuang H. J., Ho Ch. Y.: Heuristic Rule Based Phase Balancing of Distribution Systems by Considering Load Patterns, IEEE. Transactions on Power Systems 20(2), 709-716 (2005)

2. Wang K., Skiena S., Robertazzi T. G.: Phase Balancing Algorithms. Electric Power Systems Research 96, 218-224 (2013)

3. Lee C.: Feeder Reconfiguration and Capacitor Setting for Loss Reduction of Distribution Systems, Electric Power Systems Research 58(2), 97-102 (2001)

4. Chen T. H., Cherng J. T.: Optimal Phase Arrangement of Distribution Transformers Connected to a Primary Feeder for System Unbalanced Improvement and Loss Reduction Using a Genetic Algorithm. IEEE Transaction on Power Systems 15(3), 994-1000 (2000)

5. Kim H., Ko Y., Jung K.: Artificial Neural network based Feeder Reconfiguration for Loss Reduction in Distribution Systems. IEEE Transactions on Power Delivery 8(3), 1356-1366 (1993)

6. Civanlar S., Grainger J., Yin H., Lee S.: Distribution Feeder Reconfiguration for Loss Reduction. IEEE Transactions on Power Delivery 3(3), 1217-1223 (1988)

7. Dolatdar E., Soleymani S., Mozafari B.: A New Distribution Network Reconfiguration Approach using a Tree Model. World Academy of Science, Engineering and Technology 58(34), 1186 (2009)

8. Jeon Y. J., Kim J. C., Kim J. O., Lee K. Y.: An Efficient Simulated Annealing Algorithm for Network Reconfiguration in Large-Scale Distribution Systems. IEEE Transactions on Power Delivery 17(4), 1070-1078 (2002)

9. Chiang H., Jean-Jameau R.: Optimal Network Reconfiguration in Distribution Systems. IEEE Transactions on Power Delivery 5(4), 1902-1909 (1990)

10. Zhu J., Bilbro G., Chow M. Y.: Phase Balancing using Simulated Annealing. IEEE Transactions on Power Systems 14 (4), 1508-1513 (1999)

11. Bouchard D., Chikhani V., John V., Salama M.: Applications of Hopfield Neural Networks to Distribution Feeder Reconfiguration. International Forum on Applications of Neural Networks to Power Systems, 311-316 (1993)

12. Vulasala G., Sirigiri S., Thriruveedula R.: Feeder Reconfiguration for Loss Reduction in Unbalanced Distribution System Using Genetic Algorithm. International Journal of Electrical and Electronics Engineering 3(12), 754-762 (2009)

13. Lafortune M., Bouchard D., Morelli J.: Phase Swapping for Distribution System Using Tabu Search. WSEAS International Conference on Energy Planning, Energy Saving, Environmental Education, 67-71 (2007)

14. Zhu J., Chow M. Y., Zhang F.: Phase Balancing using Mixed-Integer Programming. IEEE Transactions on Power Systems 13(4), 1487-1492 (1998)

15. Fei C. G., Wang R.: Using Phase Swapping to Solve Load Phase Balancing by ADSCHNN in LV Distribution Network. International Journal of Control and Automation $7(7), 1-14(2014)$ 
16. Granada M., Gallego R., Lopez Lezama J. M.: Optimal Phase Balancing Planning for Loss Reduction in Distribution systems using a Specialized Genetic Algorithm. Ingenieria y Ciencia 8(15), 121-140 (2012)

17. Geem, Z. W., Kim, J.-H., Loganathan, G. V.: A New Heuristic Optimization Algorithm: Harmony Search. Simulation 76(2), 60-68 (2001)

18. Manjarres, D., Landa-Torres, I., Gil-Lopez, S., Del Ser, J., Bilbao M. N., SalcedoSanz S., Geem Z. W.: A Survey on Applications of the Harmony Search Algorithm. Engineering Applications of Artificial Intelligence 26(8), 1818-1831 (2013)

19. Geem, Z.W.: Music-Inspired Harmony Search Algorithm: Theory and Applications (2009)

20. Del Ser, J., Matinmikko, M., Gil-Lopez, S., Mustonen, M.: Centralized and Distributed Spectrum Channel Assignment in Cognitive Wireless Networks: A Harmony Search Approach. Applied Soft Computing 12(2), 921-930 (2012)

21. Gil-Lopez, S., Del Ser, J., Landa, I., Garcia-Padrones L., Salcedo-Sanz, S., PortillaFigueras J. A.: On the Application of a Novel Grouping Harmony Search Algorithm to the Switch Location Problem. International Conference on Mobile Lightweight Wireless Systems, 662-672 (2010) 